\title{
Microbiota of The Digestive Glands and Extrapallial Fluids of Clams Evolve Differently Over Time Depending On the Intertidal Position
}

Clément Offret ( $\nabla$ clement.offret@univ-brest.fr)

Institut Universitaire Européen de la Mer https://orcid.org/0000-0001-5786-2929

Olivier Gauthier

Institut Universitaire Européen de la Mer

Garance Despréaux

Institut Universitaire Européen de la Mer

Adeline Bidault

Institut Universitaire Européen de la Mer

Charlotte Corporeau

Univ Brest

Philippe Miner

Univ Brest

Bruno Petton

Univ Brest

Fabrice Pernet

Univ Brest

Caroline Fabioux

Institut Universitaire Européen de la Mer

Christine Paillard

Institut Universitaire Européen de la Mer

Gwenaelle Le Blay

Institut Universitaire Européen de la Mer

\section{Research Article}

Keywords: Clam, Microbiota, Digestive Gland, Extrapallial Fluids, Temporal variations

Posted Date: October 20th, 2021

DOl: https://doi.org/10.21203/rs.3.rs-976571/v1 
License: (c) (i) This work is licensed under a Creative Commons Attribution 4.0 International License. Read Full License 


\section{Abstract}

The Manila clam (Ruditapes philippinarum) is the second most exploited bivalve in the world but remains threatened by diseases and global changes. Their associated microbiota play a key role in their fitness and acclimation capacities. This study aimed at better understanding the behavior of clam digestive glands and extrapallial fluids microbiota at small, but contrasting spatial and temporal scales. Results showed that environmental variations impacted clam microbiota differently according to the considered tissue. Each clam tissue presented its own microbiota, and showed different dynamics according to the intertidal position and sampling period. Extrapallial fluids microbiota was modified more rapidly than digestive glands microbiota, for clams placed on the upper and lower intertidal position, respectively. Clam tissues could be considered as different microhabitats for bacteria as they presented different responses to small-scale temporal and spatial variabilities in natural conditions. These differences underlined a more stringent environmental filter capacity of the digestive glands.

\section{Introduction}

Host-associated microbiota are organized into trophic groups of microorganisms that interact with each other and the organ they colonize. These microorganisms come from the parents (vertical transmission) or from the immediate environment (horizontal transmission). The colonized tissues offer particular physicochemical conditions, and act as micro-habitats. Both the host and its associated microbiota contribute to the metabolic specificity of these organs. As a result, each tissue is colonized by specific microbiota communities that are both complex and dynamic [1]. Associated microorganisms contribute to varying degrees to many metabolic processes and gene expressions that can have major impacts on host nutrition, detoxification, growth, immune system development and resistance to infections [2-4]. They probably play a key role in hosts acclimation to different ecological conditions in fluctuating environments as coastal zones.

It has been shown that marine host-associated microbiota may be influenced by different biotic and abiotic factors (season, location, host age, health and genotype) [5-8]. Being filter-feeders, bivalves interact with numerous microorganisms coming from the environment. They maintain tissue-specific microbiota that differ from that of their surrounding environment [9], indicating that both organisms and tissues select the bacteria they host filters $[10,11]$. Nonetheless, these host-associated microbiota may vary spatially and temporally reflecting seasonal changes and geographic location [12-16]. Intrinsic factors, like life stage, genetic, host tissue or host taxonomy may also induce different variations in terms of microbiota composition and dynamic [17-19]. However, the mechanisms underlying the interactions in bivalve microbiota and how these ones are likely to modify their host acclimation to a changing environment are still largely unknown.

The Manila clam (Ruditapes philippinarum), is a sediment burrower that mainly ingests benthic microalgae and sedimented phytoplankton [20]. Its microbiota is influenced by environmental factors such as season [21], contaminants [22], and intertidal position [11]. They also differ among heart, gonad, 
mantle, hemolymph, gills or gut [21, 23], indicating an organ-specific microbiota. This suggests that factors may have different effects on microbiota dynamics depending on the considered tissues, and so differently affect their functions.

Almost nothing is known about clam's extrapallial fluids microbiota, although this compartment plays an important role in immunity and calcification processes [24], which could be influenced by microbial changes. Indeed, it has been previously shown that microbiota from extrapallial fluids of Eastern oyster (Crassostrea virginica) is influenced by location and season [10].

Microbiota is important in adaptation to fluctuating environment like the intertidal zone, and that bivalves are good models for that. To highlight specific responses of tissue-associated microbiota in the face of environmental small-scale variations, we investigated the dynamics of clam microbiota during the spring revival, a period that is characterized by marked environmental changes impacting the host species [25]. Animals were deployed at two tidal levels, and were sampled in February, March and April. Two tissues that are differently exposed to the environment were compared (with and without depuration), the extrapallial fluids (EF), which are at the interface between the animal and the environment, and the digestive glands (DG), an internal organ that is exposed to what is ingested. This study will provide a better understanding of the dynamic and scales of variation of microbiota associated with the DG and EF of healthy clams in natural conditions.

\section{Experimental Procedures}

\subsection{Experimental design}

The Manilla clams Ruditapes philippinarum were hatched in April 2016 and were provided by a commercial exploitation (SATMAR, France). In October 2017, clams were placed at the site IFREMER Br08 located in the Bay of Brest at Pointe du Château (48 $20^{\prime} 06.19^{\prime \prime} \mathrm{N}, 4^{\circ} 19^{\prime} 06.37^{\prime \prime}$ W, Britany, France) at two different intertidal levels corresponding to $20 \%$ (L20) and $56 \%$ (L56) of exondation time (Fig.1) in two duplicated mesh bags.

Healthy clams (i.e. without signs of Brown Ring Disease) were collected from the two intertidal levels over three consecutive days (to avoid sampling bias) in T1 (February 2018, $19.6 \pm 3.4 \mathrm{~mm}$ ), T2 (March 2018, $18.1 \pm 2.4 \mathrm{~mm}$ ) and T3 (April 2018, $19.5 \pm 2.8 \mathrm{~mm}$ ). For each sampling day, sediments were sampled in triplicate at each level, next to the clams, and seawater was collected in triplicate $2 \mathrm{~h}$ before low tide. The seawater samples (1L) were successively passed through 8 (SW8) and $0.22 \mu \mathrm{m}$ (SW022) polycarbonate filters (Whatman, USA) that were kept at $-80^{\circ} \mathrm{C}$ until DNA extraction and sequencing. For each day, clams $(n=10)$ were collected by level for bacterial microbiota analyses, and half of them $(n=5)$ were directly dissected for tissue sampling, while others $(n=5)$ were transferred to the laboratory and grouped by day and intertidal level for depuration process in order to empty the DG and reduce environmental microorganisms [26]. After 14 days in tanks $(30 \mathrm{~L})$ fed with flow-through ( $3 \mathrm{~L} / \mathrm{min}$ ) filtered seawater (10 and $5 \mu \mathrm{m}$ sand filters and UV treatment before two $1 \mu \mathrm{m}$ filters, and a second UV treatment), tissues were 
dissected and collected before DNA extraction. Tanks were cleaned every day to avoid biofilm formation and bacterial proliferation. No feed was added.

\subsection{Bacterial genomic DNA sequencing}

Microbiota analyses were performed from digestive glands (DG) and extrapallial fluids (EF) of both depurated and non-depurated clams, as detailed by Offret et al., [11]. Briefly, DG and EF were aseptically dissected from clams directly after sampling or after depuration. Bacterial genomic DNA from both clam tissues and environmental samples were extracted using the PowerLyzer PowerSoil DNA Isolation Kit (Qiagen, USA). For each sample, the DNA was amplified by PCR targeting the variable V3V4 region coding for the bacterial 16S rRNA gene using the 341F (5'-CCTACGGGNGGCWGCAG -3') and 805R (5'GACTACHVGGGTATCTAATCC-3') primers [27]. Finally, paired-end sequencing with a 300-pb read length was performed at McGill University (Génome Quebec Innovation Centre, Montréal, Qc, Canada) on a MiSeq system (Illumina).

\subsection{Bacterial microbiota analysis}

Sequences were processed with the Galaxy-supported FROGS pipeline (« Find, Rapidly, OTUs with Galaxy Solution »). This pipeline was developed in order to analyze large sets of amplicon sequences and produce abundance tables of Operational Taxonomic Units (OTUs) with their taxonomic affiliation [28].

Firstly, bacterial 16S rDNA sequences of samples were pre-process. Paired-end reads were merged using Flash [29] with 10\% mismatch tolerated. Primers and adapters were removed using cutadapt [30]. Clustering was achieved using the Swarm method with an aggregation distance (d=3) [31]. Afterwards, chimeras were detected and removed with VSEARCH (de novo UCHIME method) before applying a crosssample validation step [28, 32, 33]. Next, a relative abundance filter was applied on OTUs with a threshold of $0.005 \%$ (of global relative abundance), as recommended by Bokulich et al., [34]. Taxonomic affiliation was done on this new data by blastn+ [35], using the Silva $13216 \mathrm{~S}$ database [36] formatted to include taxonomic levels up to species [28]. For analysis, cyanobacterial and eukaryotic sequences (chloroplast and mitochondrial 16S rRNA gene from algal cells) were removed.

\subsection{Statistical analyses}

\subsubsection{Relative abundance and a-diversity}

The relative abundance of the whole bacterial microbiota communities was assessed at the order level at each period [8]. Microbiota a-diversity was assessed at the OTUs level and the rarefied richness and Shannon indices were computed. The period, level, tissue and depuration effects (and their interactions) were tested on clam's a-diversity indices. For the environmental samples, the effects of period and level (and their interaction) were tested on the sediment and the effects period and filter (and their interaction) on the seawater. For each model, the effects were tested using a linear model on Box-Cox transformed data and a Generalized Linear Model (GLM) on non-transformed data. The linear model was either a two- 
way analysis of variance (ANOVA) or an analysis of covariance (ANCOVA) with the number of sequences as a co-variable. Finally, the models with the greater adjusted $\mathrm{R}^{2}$ were kept.

\subsection{2. $\beta$-diversity}

Microbial communities $\beta$-diversity was also assessed at the OTUs level. On the one hand, the OTUs abundances tables were transformed using the Box-Cox-chord transformation with exponent 0 which corresponds to a log-chord transformation [37]. Then, the variation in microbiota composition and structure between all samples was visualized with a PCA of these transformed OTUs abundances. The effects of period, level and their interactions were tested using PERMANOVA [38] when group dispersions were homogeneous. Multivariate homogeneity of group dispersions was assessed for the DG, EF, sediments and seawater samples separately. All analyses were carried out using R3.6.2 [39], with functions from the phyloseq [40], vegan [41], emmeans [42], RAM [43] and VIM [44] packages.

\section{Results}

\subsection{Sequences analysis information}

The total number of sequences before processing was 13406 675. After processing, 7369012 sequences were kept (i.e. $54.97 \%$ ). After removing sequences affiliated to chloroplasts and samples with a number of sequences reads inferior to 3 000, 432 samples were kept: 160 DG's samples, 177 EF's samples, 41 sediment's samples and 54 seawater's samples (27 SW8 and 27 SW022). The swarm clustering produced 1255 different OTUs, which were identified in GD (1152), EF (1 201), sediments (935) and seawater (1 057 and 972 into SW8 and SW022 fractions, respectively). The mean number of sequences reads by sample was $16063 \pm 332$ for all samples: $15297 \pm 478$ for the DG, $19251 \pm 446$ for the EF, $5487 \pm 203$ for the sediments, $15917 \pm 851$ for the seawater (10 $924 \pm 601$ for SW8 and $20910 \pm$ 824 for SW022).

\section{2. a-diversity comparison for clam tissues and environmental samples}

The effect of the number of sequence reads was significant for observed richness $(F=7.6, P<0.01)$ justifying the use of a Rarefied richness for describing a-diversity patterns. Models applied to clam's tissues microbiota a-diversity indexes (rarefied richness and Shannon indices) highlighted two main factors: Depuration and Tissue (including the seawater fractions), followed by the Period (Fig.2). The Depuration induced a significant decrease of Rarefied richness $(F=4.7, P<0.01)$ and Shannon indices $(F$ $=62.2, P<0.001)$, while Tissue differences results in an average EF a-diversity that were always significantly higher than those for the GD (Rarefied richness: $F=29.4, P<0.001$; Shannon: $F=14.6, P<$ 0.001). The two seawater fractions exhibited two clearly different a-diversity, where the bacterial communities from SW8 fraction were always greater than those from the SW022 one (Rarefied richness: $F=211.9, P<0.001)$. Nonetheless, on average a-diversity of microbiota associated with the two clam tissues was always significantly lower than those of bacterial communities from sediment and SW8 (post-hoc Tukey test, $P<0.001$ ). 
The Period factor was significant for all models, except for the Shannon indices of sediment $(F=1.4, P>$ 0.05). Overall regarding both indices, average a-diversity of bacterial communities from non-depurated DG and seawater samples, significantly decreased during the studied period, notably between T1 and T3 (post-hoc Tukey test, $P<0.001$ ). In the other hand, non-depurated EF and sediment alpha diversities significantly increased between $\mathrm{T} 1$ and $\mathrm{T} 3$.

\subsection{Order level bacterial diversity from clam tissues and environmental samples}

Based on the 30 most abundant bacterial orders, DG, EF, sediment and seawater fractions showed clearly distinct bacterial communities in terms of both abundance and taxa composition (Fig.3). The two fractions of seawater (SW8 and SW022) were dominated by the orders Rhodobacterales (55-70 \% in SW022 and 20-30 \% in SW8) and Flavobacteriales (12-15\% in SW022 and 10-22 \% in SW8). However, their composition differed for less abundant orders (inferior to $6 \%$ of total abundance). Indeed, SW022's bacterial community was mostly composed of Oceanospirillales and Cellvibrionales, whereas SW8's bacterial community was more diverse with Cellvibrionales, Desulfobacterales, Campylobacterales, Pirellulale, Rhizobiales, Verrucomicrobiales, Clostridiales and Myxococcales present in low abundance (inferior to $5 \%$ ). Sediment bacterial communities were mostly composed of numerous few abundant orders, accounting for more than $25 \%$ of the total abundance, while the most abundant orders were mainly affiliated to Desulfobacterales (13-19\%), Actinomarinales (6-8\%), Campylobacterales (5-7\%), Microtrichales (5-7\%) and Rhizobiales (4-6\%).

The non-depurated clams, DG microbiota was mostly composed in a decreasing order of contribution, Rickettsiales, Mycoplasmatales, Diplorickettsiales, Spirochaetales, Pirellulales, Clostridales,

Oceanospirillales and Flavobacteriales. DG microbiota from clams sampled in T1 was characterized by a lower relative abundance of Rickettsiales and a higher relative abundance of Spirochaetales and Clostridiales, while higher relative abundances of Campylobacterales and Flavobacteriales were observed respectively in T2 and T3. One of the most abundant order found in DG microbiota, the Diplorickettsiales, drastically decreased in T3 compared to T1 and T2. In depurated clams, the DG microbiota was globally composed of the same orders as non-depurated clams, but in different abundances. The main changes were observed for Spirochaetales, increasing from around $6 \%$ to $20 \%$ between the non-depurated and depurated clams, while Pirellulales abundances were divided by 7 in depurated clams.

Although it harbored some taxa (at the order level) in common, EF microbiota of non-depurated clams was clearly different from that of the DG's. It was mainly composed of Oceanospirillales, Spirochaetales, Rickettsiales, Flavobacteriales, Sneathiellales, Campylobacterales, and also Rhodobacterales, Francisellales, Verrucomicrobiales, Cellvibrionales and Alteromonadales, those last clearly contributing to the differences with DG microbiota. In T1, the EF microbiota was characterized by a higher relative abundance of Rickettsiales and Oceanospirillales and a lower abundance of Alteromonadales, Sneathiallales and Rhodobacterales. A higher relative abundance of Campylobacterales and Flavobacteriales were observed in T2 and T3, respectively. In EF microbiota from depurated clams, the same orders were globally found than in non-depurated clams but in different abundances, notably for 
Alteromonadales, Francisellales and Pseudomonadales which were present in much higher abundances, whereas Flavobacteriales and Rhodobacterales were reduced.

\subsection{Factors influencing Beta diversity}

From this point on, results are those of analyses conducted at the OTU's level. PCA (Fig.4) allow clearly discriminating sample matrix (clams vs sediment and seawater on PC1), seawater fractions (SW8 vs SW022 on PC2) and clam tissues (EF vs DG on PC2). The SW8 bacterial community was more similar to that of sediments, whereas the one from SW022 was more similar to that of the EF.

Homogeneity of variances was previously tested for each sample grouping, revealing that multivariate dispersion was not homogenous (Fig.5) for both $D G(F=17.333, P<0.001,999$ permutations) and EF tissues ( $F=6.893, P=0.001,999$ permutations). Using the betadisper function, the DG microbiota had a higher heterogeneity of group dispersions than EF. The heterogeneity of dispersions occurred between the depurated samples of L56 in T3 and all the groups in T1 (Fig. 5A). It also occurred between the nondepurated samples of L20 in T2 and all the groups in T2. The dispersions were also heterogeneous between T1 and T3. In the case of the EF's microbiota (Fig. 5B), the heterogeneity of dispersions implied three groups of clams: the depurated at L20 in T1, the non-depurated at L56 in T2 and all the depurated in T3. The L20 levels of T2 and T3 seemed more similar to T1 (L20 and L56) than to L56. For the depurated clams, all clusters were overlapping, indicating a more similar community over the three periods. However, L56 and L20 of the three periods tended to pull away from each other and the centroid, indicating differences between levels.

Regarding the DG bacterial microbiota (Fig.5a), a high heterogeneity of dispersion, which increased between T1 and T3, was observed. However, it did not prevent us from seeing the dynamic of the microbiota. A clear distinction between depurated and non-depurated clam's microbiota was observed along the first axis of the PCA for both levels (L20 \& L56) in T1 and T2, which was much less the case in T3. For the non-depurated clams, the DG bacterial microbiota in T1 and T2 were very close regardless of the levels, no period or level effects were observed. It is only in T3 that the DG bacterial microbiota detached (especially for L20) from T1 and T2, and that a slight level effect appeared. For the depurated clams, the DG microbiota were more similar between the three periods. Moreover, in that case the level effect was more pronounced in T2 than in T3.

For the EF bacterial microbiota (Fig.5b), compared to the DG microbiota, the distinction between depurated and non-depurated was clearer along the first axis, as clusters did not overlap in the PCA regardless of levels and periods. The EF microbiota behaved differently from the DG ones. In T1, no level effect was visible (as for the DG), but in T2 and T3 the level effect becomes more important than the period effect, with a clear distinction between levels regardless of the time period. The L56 levels were clearly distinct from the other ones in both T2 and T3.

The PCA representing the sediments communities (Fig.5c) showed a level effect but no period effect. Only the L56 samples in T1 appeared to have a distinct microbiota from other L56 samples. Multivariate 
analysis of variance on the Box-Cox transformed and standardized environmental data reveals a period effect $\left(F=1.77, P=0.025, R^{2}\right.$ adj $\left.=0.069\right)$, the level $\left(F=9.25, P=0.001, R^{2} a d j=0.18\right)$ and their interaction $\left(F=1.59, P=0.041, R^{2} a d j=0.062\right)$. The seawater's bacterial community (Fig.5d) was clearly different between the three periods and the two filters. The heterogeneity of dispersion between the groups did not allow a multivariate analysis of variance $(F=3.6108, P=0.037,999$ permutations).

\section{Discussion}

In this study, we investigated the diversity and the temporal dynamic of the DG and EF microbiota from Manilla clams placed on two intertidal levels in the foreshore during spring revival. Results brought more information regarding specificity of clam tissue microbiota, while both small spatial and temporal scales significantly contributed to the shaping of the DG and EF microbiota. The observed differences were attributed in a decreasing order to the depuration process, the tissue, the sampling period and finally to the intertidal level. Furthermore, different dynamics were clearly observed depending on the tissue and intertidal level.

\subsection{The environmental contribution to the clam holobiont}

Regarding clam microbiota, both DG and EF were clearly different from the environment, although the bacterial communities in the surrounding seawater and sediments contributed to the microbiota shaping. Indeed, EF microbiota was much closer to environmental bacterial communities than DG microbiota, especially from the $0.22-8 \mu \mathrm{m}$ seawater fraction, suggesting a higher proximity between EF and seawater (free and small particles associated bacteria) than with the digestive glands. OTUs present in the clams DG microbiota, such as Rickettsiales, Mycoplasmatales, Diplorickettsiales, Spirochaetales, Oceanospirillales and Flavobacteriales, as commonly found in clams DG [11,22], with Mycoplasmatales and Rickettsiales attributed to the core members [22]. EF microbiota harbored a high abundance of taxa orders such as Rhodobacterales, similar to those found in seawater but not in the DG. This is probably the result of a direct exchanges between seawater and EF, maybe through the pallial line, possibly associated with less stringent EF physical and chemical conditions for the seawater bacteria than in the DG. To the author's knowledge, EF microbiota of Manila clams has never been described so far. However, Rickettsiales and Flavobacteriales were already found in high abundance in EF microbiota from another bivalve species, the Eastern Oyster Crassostrea virginica [10].

\subsection{Tissue-specific of clam microbiota dynamics}

This study revealed a different response of the two-tissue microbiota over time. Even if both DG and EF microbiota showed a strong temporal variability in terms of a-diversity, EF's a-diversity significantly increased over time in their natural environment, whereas that of the DG's decreased. Indeed, organ microbiota are shaped by the organ's physiological role, notably digestion and shell mineralization for DG and EF, respectively (Wilbur and Saleuddin, 1983; Allam and Paillard, 1998). On the one hand, the Manila clam's different organs are known to be bacterial niches with unique features [21], and so they could be considered as different micro-habitats. On the other hand, this could be linked to environmental 
variations, such as temperature and dissolved oxygen which have already been shown to impact $\mathrm{EF}$ microbial composition of Pacific Oysters [10]. Regarding DG microbiota, a higher a-diversity has been described from hepatopancreas microbiota of the Manilla clams sampled in winter than in summer [22]. We hypothesize that the observed decrease of bacterial a-diversity in clam DG, could be caused by presence of other water associated microorganisms such as micro-eukaryotes.

In addition to the temporal heterogeneity, a tidal level effect has also been observed for both DG and EF, but their dynamics were very different. The greater effect of the intertidal level on EF microbiota in T2 and T3 may be explained by a greater heterogeneity of environmental conditions in L56 since the immersion period was shorter. Microbiota from bivalve fluids seemed to be particularly sensitive to the sampling zone as it was shown for hemolymph's microbiota from the Pacific Oyster [47]. These results seem to hint that EF microbiota are more impacted by environmental variations when they are placed in the upper part of the foreshore. On the contrary, DG microbiota were more impacted by the period when they were placed in the lower part of the foreshore (L20), which was under a greater influence of sea water and feeding sources. So, depending on the considered tissue, microbiota dynamics vary with intertidal position.

\subsection{GD are more stringent environmental filters than $E F$}

Analysis of $\beta$-diversity components (data not shown) also revealed distinct variations between the twotissue microbiota. EF bacterial microbiota differences between individuals were mostly driven by species replacement for the all three sampling periods. It is thus hypothesized that EF reached maximum niche occupancy. On the other hand, DG bacterial microbiota $\beta$-diversity was mostly driven by richness difference, except for clams sampled in T1. These results highlight the possibility that OTUs richness might be more constrained in EF and less so in DG.

In addition to the fact that a-diversity was higher in the EF microbiota, depuration process more affected $\mathrm{EF}$ than GD may illustrate that EF microbiota is composed of more transient microorganisms coming from the surrounding sea water. The analysis of $\beta$-diversity revealed a higher dispersion in DG microbiota compared to EF microbiota, illustrating a higher within-tissue diversity in the DG. All these results revealed that DG is a potentially more stringent environmental filter than EF.

In conclusion, this study has shown that environmental variations may have a strong influence on clam microbiota, through impact on both digestive gland and extrapallial fluids associated bacteria. DG and EF presented their own microbiota specificity in terms of abundance, richness and dynamics. Furthermore, clam's tissue could be considered as distinct habitats from the bacterial OTUs point of view, as they presented different responses to either small-scale temporal and spatial variabilities or to a depuration process, illustrating that they their selection processes differ. These differences underlined that DG could be a more stringent micro-habitat probably linked to its particular physico-chemical conditions. This study helps to have a better understanding of the Manila Clam's microbiota dynamic in the face of environmental variation, and especially of the EF microbiota which is still very poorly known. 


\section{Declarations}

Declarations: Not applicable.

Funding: This work was supported by the ISblue project, Interdisciplinary graduate school for the blue planet (grant number ANR-17-EURE-0015) and co-funded by a grant from the French government under the program 'Investissements d'Avenir'. The Region Bretagne SAD (2017, Stratégie d'Attractivité Durable) contributed to this study through the postdoctoral fellowship of Clement Offret. This work was also supported by the HORIZON2020 project 'Preventing and mitigating farmed bivalve disease' VIVALDI (grant number 678589).

Conflict of interest: The authors declare that the research was conducted in the absence of any commercial or financial relationships that could be construed as a potential conflict of interest.

Availability of data and material: The sequence data have been deposited in the NCBI database under BioProject ID PRJNA748558.

Authors' contributions: C.O., G.D., G.L.B. and O.G. wrote the manuscript, and all authors commented on the final version. Experiments, microbiota analyses, results interpretation, C.O.; microbiota analyses, results interpretation, G.D.; statistical analyses, O.G. and GD; Project and experimental design, oyster and clam dissections, A.B., P.M., B.P., C.C., F.P., C.F., Project and experimental design, oyster and clam dissections and funding acquisition C.P.; Project supervision and experimental design, field monitoring, oyster and clam dissections, results interpretation and funding acquisition, G.L.B.

\section{Acknowledgements}

The authors wish to thank Bruno Petton, Elodie Fleury, Christophe Lambert, Nelly Le Goic, Morgan Perennou, Christine Dubreuil, Valerian Le Roy, Claudie Quere, Amelie Segarra, Alexandra Rahmani, Elyne Dugeny, Morgan Smith, Lizenn Delisle, Veronique Loiseau and Amandine Morot for their help with sampling preparation and dissections of bivalves, and Dominique Hervio for her help with water filtration. Thanks to Stephane Pouvreau and the Marine environmental station database of Daoulas bay for their essential help (https://www.seanoe.org/data/00314/42493/).

\section{References}

1. Weissenbach J, Sghir A (2016) Microbiotes and Metagenomics. Medecine sciences: M/S 32:937943

2. Rawls JF, Samuel BS, Gordon JI (2004) Gnotobiotic zebrafish reveal evolutionarily conserved responses to the gut microbiota. Proceedings of the National Academy of Sciences 101:4596-4601

3. Rosenberg E, Koren O, Reshef L et al (2007) The role of microorganisms in coral health, disease and evolution. Nat Rev Microbiol 5:355-362. https://doi.org/10.1038/nrmicro1635 
4. Dishaw LJ, Cannon JP, Litman GW, Parker W (2014) Immune-directed support of rich microbial communities in the gut has ancient roots. Developmental Comparative Immunology 47:36-51

5. Gil-Agudelo DL, Myers C, Smith GW, Kim K (2006) Changes in the microbial communities associated with Gorgonia ventalina during aspergillosis infection. Dis Aquat Organ 69:89-94

6. Koren O, Rosenberg E (2006) Bacteria associated with mucus and tissues of the coral Oculina patagonica in summer and winter. Appl Environ Microbiol 72:5254-5259

7. Littman RA, Willis BL, Pfeffer C, Bourne DG (2009) Diversities of coral-associated bacteria differ with location, but not species, for three acroporid corals on the Great Barrier Reef. FEMS Microbiol Ecol 68:152-163

8. Chen C-P, Tseng C-H, Chen CA, Tang S-L (2011) The dynamics of microbial partnerships in the coral Isopora palifera. The ISME Journal 5:728-740

9. Harris JM (1993) The presence, nature, and role of gut microflora in aquatic invertebrates: A synthesis. Microb Ecol 25:195-231. https://doi.org/10.1007/BF00171889

10. Sakowski EG (2015) The microbiome of the eastern oyster, Crassostrea virginica, in health and disease. Thesis, University of Delaware

11. Offret C, Paulino S, Gauthier $O$ et al (2020) The marine intertidal zone shapes oyster and clam digestive bacterial microbiota. FEMS Microbiol Ecol 96:. https://doi.org/10.1093/femsec/fiaa078

12. Lokmer A, Wegner KM (2014) Hemolymph microbiome of Pacific oysters in response to temperature, temperature stress and infection. ISME J 9:670-682. https://doi.org/10.1038/ismej.2014.160

13. Cúcio C, Engelen AH, Costa R, Muyzer G (2016) Rhizosphere microbiomes of European seagrasses are selected by the plant, but are not species specific. Frontiers in microbiology 7:440

14. Sharp KH, Pratte ZA, Kerwin AH et al (2017) Season, but not symbiont state, drives microbiome structure in the temperate coral Astrangia poculata. Microbiome 5:120

15. Woo S, Yang S-H, Chen H-J et al (2017) Geographical variations in bacterial communities associated with soft coral Scleronephthya gracillimum. PLOS ONE 12:e0183663. https://doi.org/10.1371/journal.pone.0183663

16. Vijayan N, Lema KA, Nedved BT, Hadfield MG (2019) Microbiomes of the polychaete Hydroides elegans (Polychaeta: Serpulidae) across its life-history stages. Mar Biol 166:19. https://doi.org/10.1007/s00227-019-3465-9

17. Trabal Fernández N, Mazón-Suástegui JM, Vázquez-Juárez R et al (2014) Changes in the composition and diversity of the bacterial microbiota associated with oysters (Crassostrea corteziensis, Crassostrea gigas and Crassostrea sikamea) during commercial production. FEMS Microbiol Ecol 88:69-83. https://doi.org/10.1111/1574-6941.12270

18. Pierce ML, Ward JE (2019) Gut Microbiomes of the Eastern Oyster (Crassostrea virginica) and the Blue Mussel (Mytilus edulis): Temporal Variation and the Influence of Marine Aggregate-Associated. Microbial Communities mSphere 4:e00730-e00719. /msphere/4/6/mSphere730-19.atom. https://doi.org/10.1128/mSphere.00730-19 
19. King WL, Siboni N, Kahlke T et al (2020) Regional and oyster microenvironmental scale heterogeneity in the Pacific oyster bacterial community. FEMS Microbiol Ecol 96:. https://doi.org/10.1093/femsec/fiaa054

20. Simons AL, Churches N, Nuzhdin S (2018) High turnover of faecal microbiome from algal feedstock experimental manipulations in the Pacific oyster (Crassostrea gigas). Microb Biotechnol 11:848858. https://doi.org/10.1111/1751-7915.13277

21. Meisterhans G, Raymond N, Girault E et al (2016) Structure of Manila Clam (Ruditapes philippinarum) Microbiota at the Organ Scale in Contrasting Sets of Individuals. Microb Ecol 71:194-206. https://doi.org/10.1007/s00248-015-0662-z

22. Milan M, Carraro L, Fariselli $P$ et al (2018) Microbiota and environmental stress: how pollution affects microbial communities in Manila clams. Aquat Toxicol 194:195-207. https://doi.org/10.1016/j.aquatox.2017.11.019

23. Leite L, Jude-Lemeilleur F, Raymond $\mathrm{N}$ et al (2017) Phylogenetic diversity and functional characterization of the Manila clam microbiota: a culture-based approach. Environ Sci Pollut Res 24:21721-21732. https://doi.org/10.1007/s11356-017-9838-z

24. Allam B, Pales Espinosa E (2016) Bivalve immunity and response to infections: Are we looking at the right place? Fish Shellfish Immunol 53:4-12. https://doi.org/10.1016/j.fsi.2016.03.037

25. Lavaud R, Artigaud S, Le Grand F et al (2018) New insights into the seasonal feeding ecology of Pecten maximus using pigments, fatty acids and sterols analyses. Mar Ecol Prog Ser 590:109-129

26. Lee R, Lovatelli A, Ababouch L (2008) Bivalve depuration: fundamental and practical aspects. Food and Agriculture Organization of the United Nations

27. Herlemann DP, Labrenz M, Jürgens $K$ et al (2011) Transitions in bacterial communities along the $2000 \mathrm{~km}$ salinity gradient of the Baltic Sea. ISME J 5:1571-1579. https://doi.org/10.1038/ismej.2011.41

28. Escudié F, Auer L, Bernard M et al (2018) FROGS: Find, Rapidly, OTUs with Galaxy Solution. Bioinformatics 34:1287-1294. https://doi.org/10.1093/bioinformatics/btx791

29. Magoč T, Salzberg SL (2011) FLASH: fast length adjustment of short reads to improve genome assemblies. Bioinformatics 27:2957-2963

30. Martin M (2011) Cutadapt removes adapter sequences from high-throughput sequencing reads. EMBnet journal 17:10-12

31. Mahé F, Rognes T, Quince C et al (2014) Swarm: robust and fast clustering method for ampliconbased studies. PeerJ 2:e593

32. Edgar RC, Haas BJ, Clemente JC et al (2011) UCHIME improves sensitivity and speed of chimera detection. Bioinformatics 27:2194-2200

33. Rognes T, Flouri T, Nichols B et al (2016) VSEARCH: a versatile open source tool for metagenomics. PeerJ 4:e2584. https://doi.org/10.7717/peerj.2584 
34. Bokulich NA, Subramanian S, Faith JJ et al (2013) Quality-filtering vastly improves diversity estimates from Illumina amplicon sequencing. Nat Methods 10:57-59.

https://doi.org/10.1038/nmeth.2276

35. Camacho C, Coulouris G, Avagyan V et al (2009) BLAST+: architecture and applications. BMC Bioinform 10:421

36. Quast C, Pruesse E, Yilmaz P et al (2012) The SILVA ribosomal RNA gene database project: improved data processing and web-based tools. Nucleic acids research 41:D590-D596

37. Legendre P, Borcard D (2018) Box-Cox-chord transformations for community composition data prior to beta diversity analysis. Ecography 41:1820-1824

38. McArdle BH, Anderson MJ (2001) Fitting Multivariate Models to Community Data: A Comment on Distance-Based Redundancy Analysis. Ecology 82:290-297. https://doi.org/10.1890/00129658(2001)082[0290:FMMTCD]2.0.C0;2

39. Team RC (2018) R: A language and environment for statistical computing. R Foundation for Statistical Computing, Vienna, Austria. 2012. URL http://www R-project org

40. McMurdie PJ, Holmes S (2013) phyloseq: an R package for reproducible interactive analysis and graphics of microbiome census data. PloS one 8:e61217

41. Oksanen J, Blanchet FG, Friendly M et al (2019) vegan: Community Ecology Package

42. Lenth R, Buerkner P, Herve M et al (2020) emmeans: Estimated Marginal Means. aka Least-Squares Means

43. Chen W, Simpson J, Levesque CA (2016) RAM: R for amplicon-sequencing-based microbial-ecology. $\mathrm{R}$ package version 1

44. Kowarik A, Templ M (2016) Imputation with the R Package VIM. J Stat Softw 74:1-16

45. WILBUR KM, Saleuddin ASM (1983) Shell formation. In: The mollusca. Elsevier, pp 235-287

46. Allam B, Paillard C (1998) Defense factors in clam extrapallial fluids. Diseases of Aquatic Organisms 33:123-128. https://doi.org/10.3354/dao033123

47. Lokmer A, Goedknegt MA, Thieltges DW et al (2016) Spatial and Temporal Dynamics of Pacific Oyster Hemolymph Microbiota across Multiple Scales. Front Microbiol 7:. https://doi.org/10.3389/fmicb.2016.01367

\section{Figures}




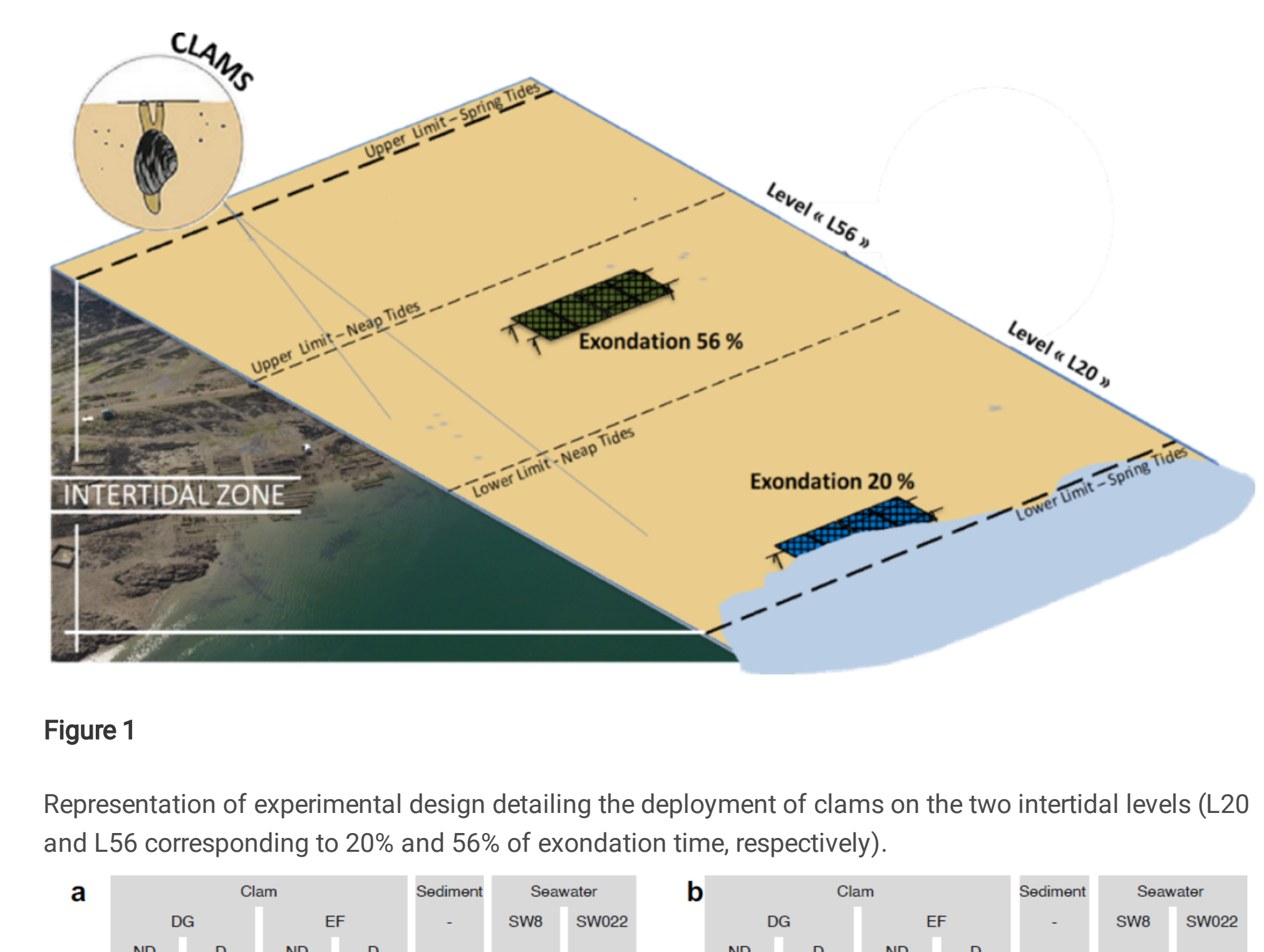

Figure 1

Representation of experimental design detailing the deployment of clams on the two intertidal levels (L20 and L56 corresponding to $20 \%$ and $56 \%$ of exondation time, respectively).
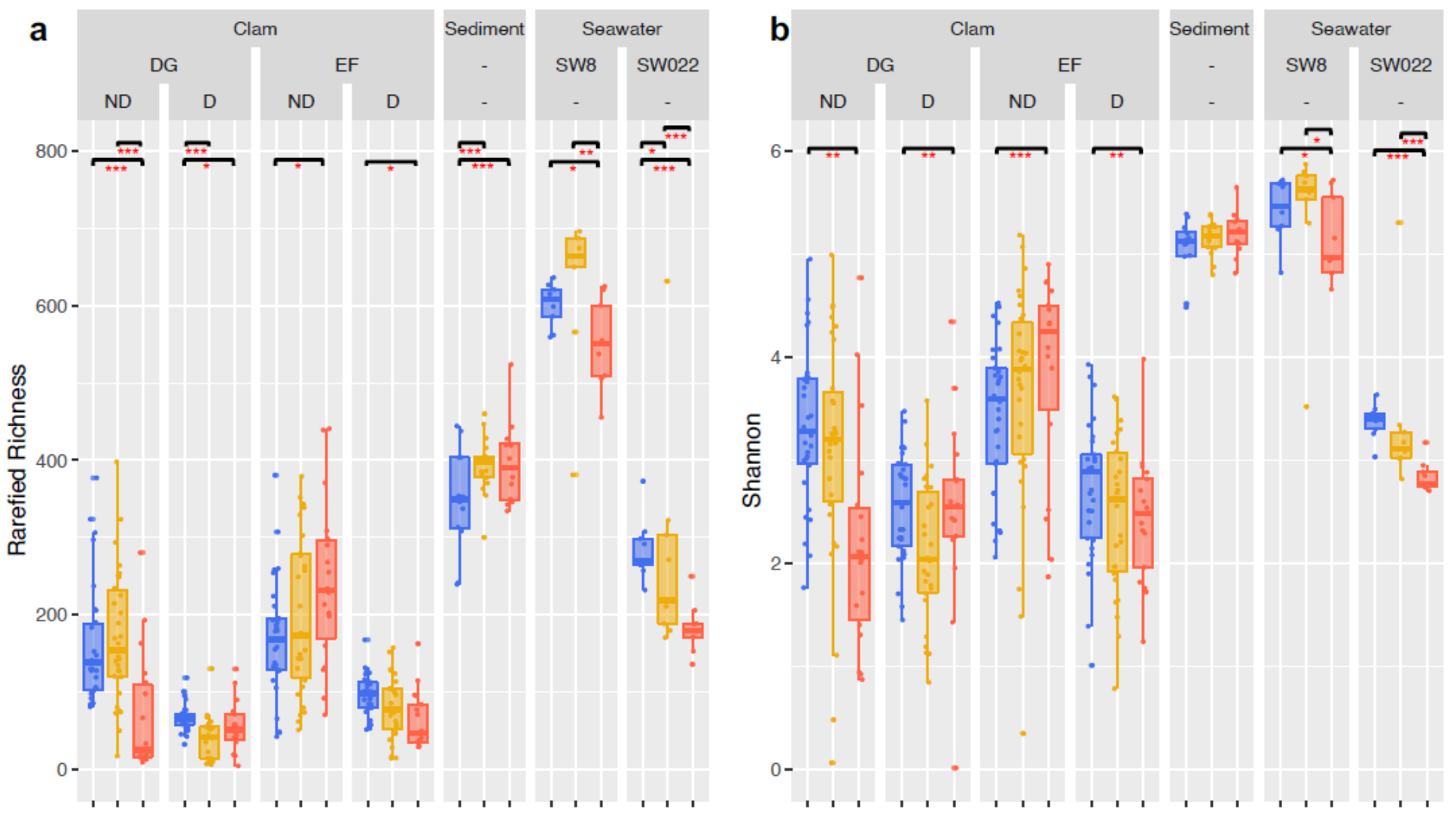
Figure 2

a-diversity indices (a Rarefied richness, b Shannon) of clam tissues microbiota and bacterial communities from sediment and seawater along the three-sampling periods, T1 (blue), T2 (yellow) and T3 (red). Samples were divided according to the significant factors (Tissue, Depuration, Period). Asterix indicate significant pairwise comparisons between the three periods $(*, P<0.05$; $* *, P<0.01$; ***, $\mathrm{P}<$ 0.001 , post-hoc Tukey test).

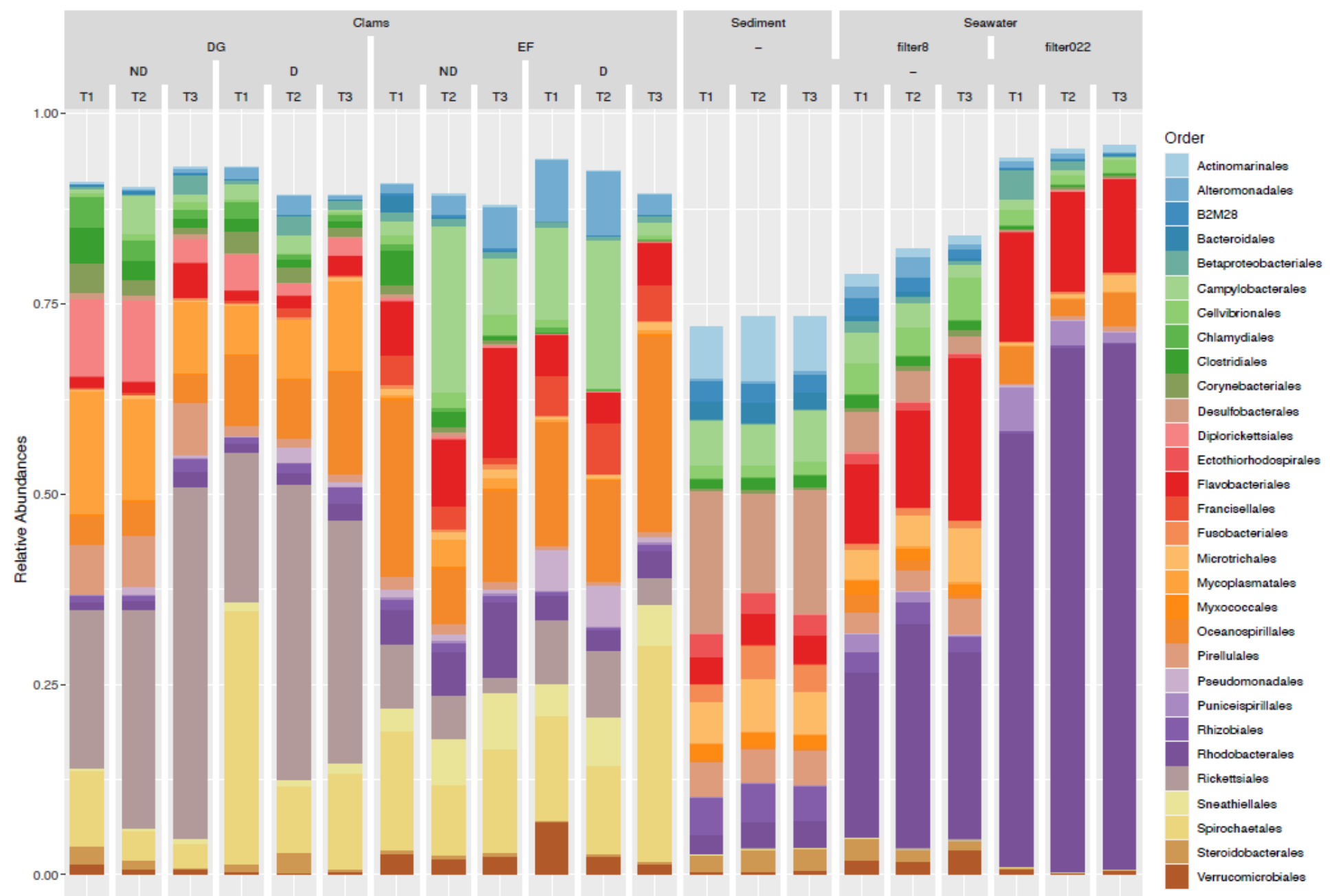

\section{Figure 3}

Relative abundance of the 30 most abundant bacterial orders in digestive glands (DG) and extrapallial fluids (EF) in non-depurated (ND) and depurated (D) clams, and found in sediments (Sed), $8 \mu \mathrm{m}$ (SW8) and $0.22 \mu \mathrm{m}$ (SW022) seawater fractions, sampled at T1, T2 or T3. 


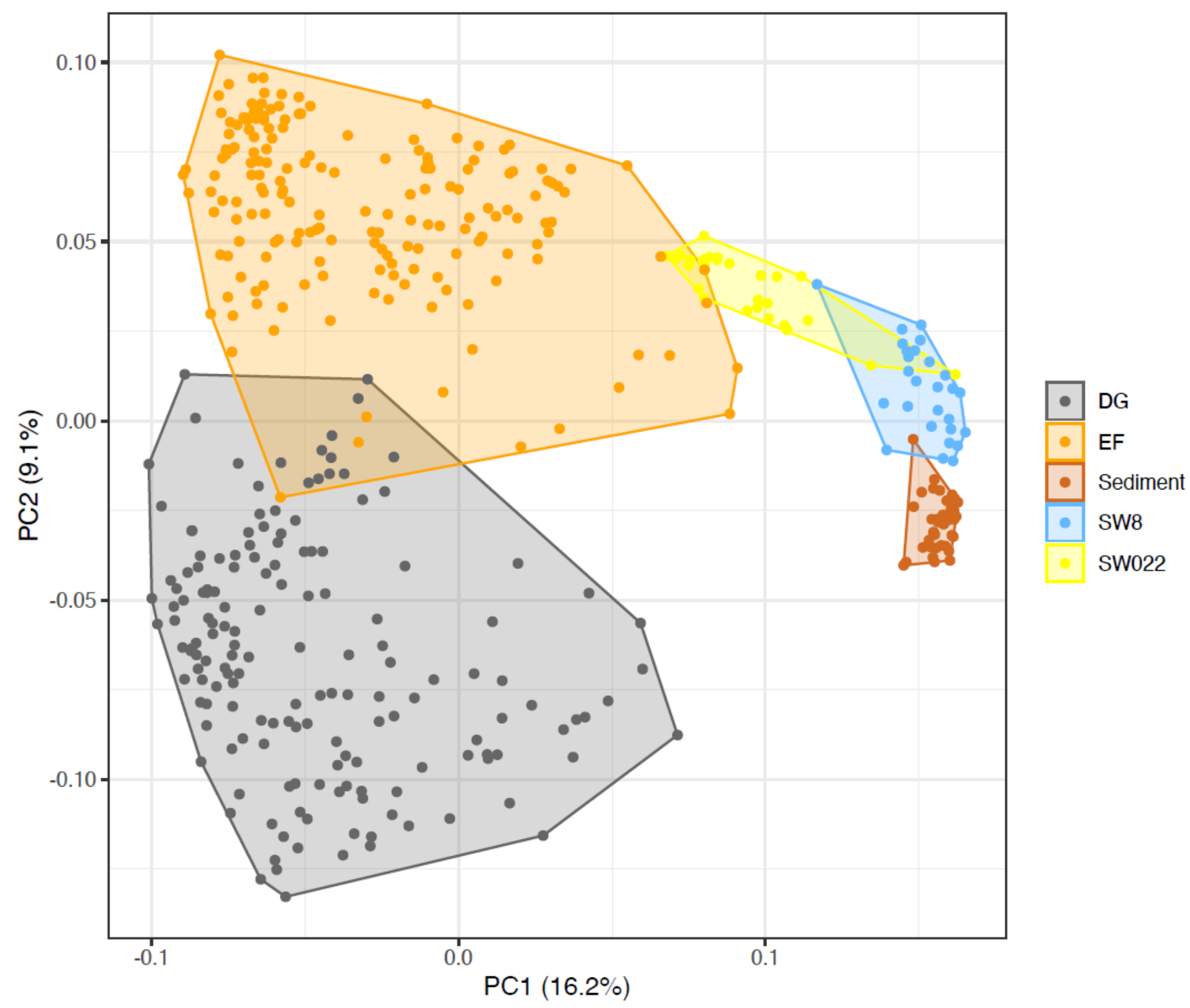

Figure 4

Distance biplot of the principal component analysis of the log-chord transformed OTUs abundance of the bacterial communities of the DG (black), EF (orange), sediments (brown), SW8 (blue) and SW022 (yellow). The two axes represent $25.3 \%$ of total variance. 

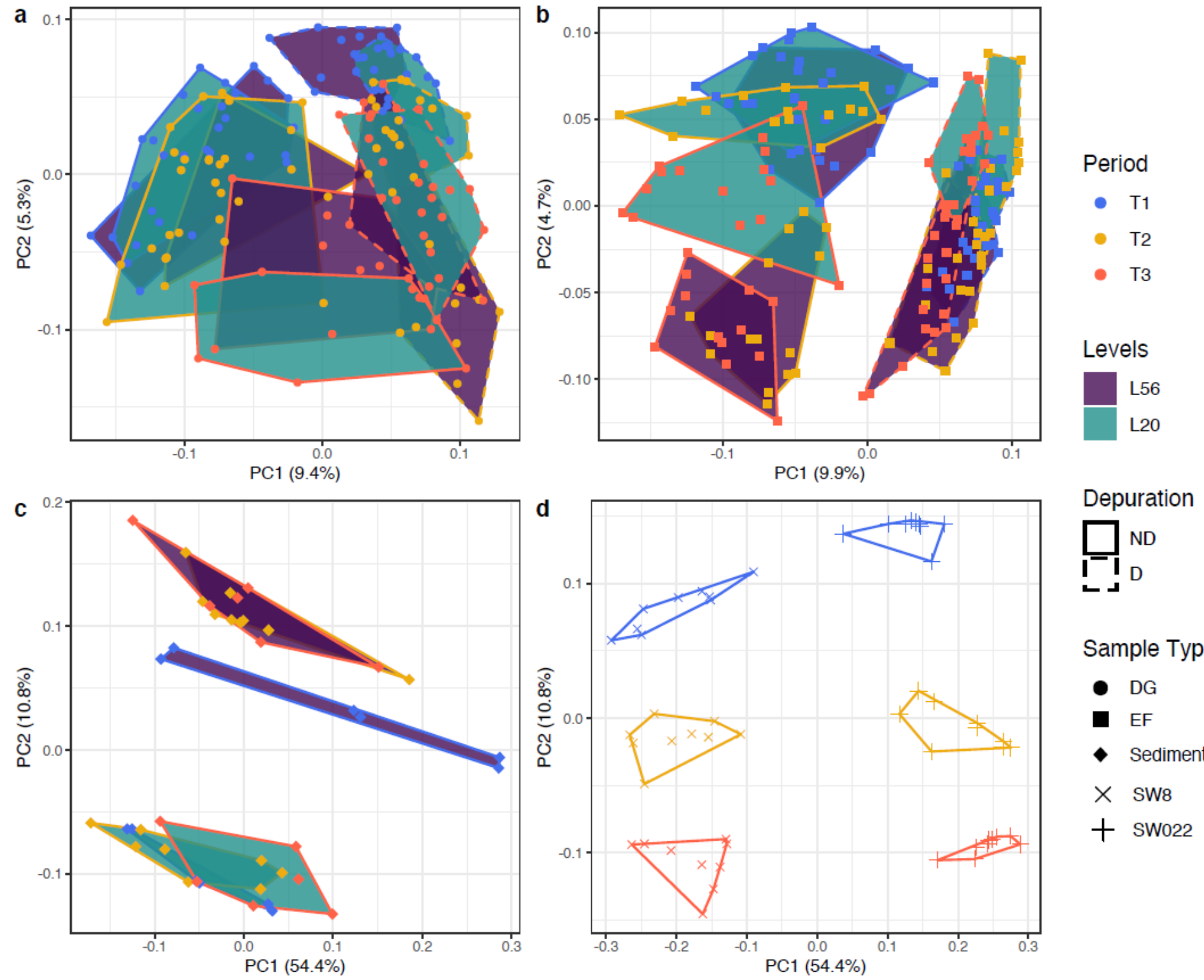

\section{Figure 5}

Distance biplots of the principal component analysis of the log-chord transformed OTUs abundance of the bacterial communities of the DG (a), EF (b), Sediment (c) and Seawater (d). Symbol colors and shapes in legend separating the sampling period combined to intertidal level, and the depuration process or seawater fractions. 